\title{
TRANSOBSTURATOR TAPE (TOT) PROCEDURE: THE IPOH EXPERIENCE
}

\section{Nusee $Z^{1}$, Naidu $A^{2}$, Ranjudham $\mathbf{S N}^{2}$, Ismail $\mathbf{H}^{1}$}

1 Division of Urogynecology, Department of Obstetrics and Gynecology, Kulliyyah of Medicine, International Islamic University, Jalan Hospital Campus, Kuantan, Pahang, Malaysia

2 Obstetrics \& Gynaecology Department, Hospital Ipoh, Perak, Malaysia

\begin{abstract}
The use of transobturator tape (TOT) for the treatment of stress urinary incontinence (SUI) has gained popularity since it was introduced in 2001. Only few studies have reported that TOT is superior in safety and efficacy when compared to tension free vaginal tape (TVT). This case-series study was conducted to investigate the safety and efficacy of the TOT procedure in patients at Ipoh Hospital. A cross sectional study on patients who underwent TOT procedures from Jan 2006 to Jun 2008 was conducted. Patients were followed-up 1 month, 3 month, 12 month and annually thereafter. Data were retrieved from patients' records based on the standard pre-operative assessment sheet and follow up record. Interviews were then made via telecommunication to assess the patients' progression and satisfaction using a validated questionnaire. Fiftytwo subjects $(n=52)$ were recruited. The mean age was $54.2 \pm 10.6$ years with the median follow up time of 19 months. Majority of cases ( $n=46 ; 88.5 \%$ ) had concomitant pelvic organ prolapse (POP), while only $2(3.8 \%)$ had an isolated stress urinary incontinence (SUI). Pre-operatively, only 16 patients $(30.8 \%)$ were diagnosed with mixed urinary incontinence (MUI) and 24 patients (46.2\%) had over active bladder symptoms (OAB) which include increased frequency and urgency. The objective cure rate for SUI was $42(80.7 \%), 3(5.8 \%)$ failed while $7(13.5 \%)$ improved. Complications were limited to minor conditions and was only observed in four patients: one case $(1.9 \%)$ of acute urinary retention, three $(5.7 \%)$ had prolonged indwelling catheter more than 3 days and one $(1.9 \%)$ each for mesh erosion, hematoma, urinary tract infection (UTI) and wound infection. Cure rate for MUI, frequency and urgency were $37.4 \%, 70.8 \%$ and $54.2 \%$ respectively. De novo urgency was noted in three $(n=3: 5.7 \%)$ cases while $(n=3: 5.7 \%)$ required revision of the tape. Forty-nine women $(n=49: 94.2 \%)$ were satisfied with the procedure. TOT procedures significantly improve OAB symptoms with high patient's acceptance rate and no serious operative complication.
\end{abstract}

KEYWORDS: transobturator tape, stress urinary incontinence, pelvic organ prolapse

\section{Correspondence:}

Dr. Zalina Nusee

Department of Obstetrics \& Gynaecology, Kulliyyah of Medicine

International Islamic University Malaysia

Bandar Indera Mahkota

25200 Kuantan, Pahang, Malaysia

E mail: drzalina@iiu.edu.my

Tel: 0199880506 


\section{Introduction}

Treatment for stress urinary incontinence (SUI) is fairly well established, due to its common occurrence amongst women. As the result of complications observed following previous urethral suspension surgeries, which includes acute urinary and chronic voiding issues, improvements were later introduced to this technique which reduced complications dramatically. This was achieved by creating a support between the mid-urethra and the anterior vaginal wall [1]. Referring to the first description of the TVT procedure by Ulmsten and Petros in 1995 [2], the minimally invasive mid urethral sling (MUS) was widely adopted as the first line surgical treatment for female SUI [3]. Although TVT has a high success rate ranging from 84 to $95 \%$ [1 - 4], there are concerns regarding its operative safety in relation to bowel and major blood vessel injuries, bladder and urethral perforation [3], and post-operative voiding difficulties or urgencies [5 - 7].

In 2001, a new surgical technique of mid-urethral prosthetic suspension called the transobturator tape (TOT, Mentor-Porgès, Le Plessis-Robinson, France) was introduced in an attempt to reduce the intraoperative complications related to traversing the retropubic space [8]. Although systematic reviews of reported cases using these procedures have demonstrated the efficacy of MUS, $5-20 \%$ of patients reports of surgical failure resulting in the persistence or recurrence of SUI [9]. TOT appears to reduce the risk of complications reported in TVT procedures while maintaining similar cure rates of SUI $[8,10]$. In addition, MUS is a much more cost effective as compared to colposuspension [11].

TVT was first introduced in Ipoh and Taiping Hospital in early 2005. The reported cure rate was $96 \%$ (Kamal et al -unpublished data) with intra-operative and post-operative complications of $13 \%$ and $34.8 \%$, respectively. In view of high morbidity, the practice was changed to TOT in 2006 which had become the standard practice then.

This study was conducted to determine the cure rate and patient's satisfaction level of TOT procedure, to determine the outcome of lower urinary tract (LUT) symptoms post operatively and to describe the postoperative complications pertaining to TOT procedure.

\section{Materials and Method}

Patients who underwent TOT procedure, performed by a single experienced surgeon, between January 2006 and June 2008 in Ipoh Hospital were reviewed. Hospital ethics committee approval was obtained prior to the study. Surgical database was retrieved from the hospiral computer and was assessed. Prospectively, the subjects were followed up via telephone interview and data were captured using a modified questionnaire. Surgery was only offered if conservative therapy was unsuccessful in all urodynamic proven SUI. Women with concomitant problems (e.g. utero-vaginal prolapse, anterior and posterior vaginal wall prolapse) were also included in the study except those with predominant urgency incontinence (UUI) and urodynamic bladder overactivity (DO).

Patients recruited for this study underwent history and pelvic examinations, urine dipstick tests and cultures, pre-operative urodynamic examination (cystometry and uroflowmetry), and post-void residual volume (PVR) measurement by bladder scan. A standard pre-operative questionnaire related to lower urinary tract symptoms (LUT) were conducted (attachment 1).

The TOT procedure performed followed the technique described by Delorme using the MONARC (American Medical Systems) Uratape:

(i) $20 \mathrm{~mm}$ incision was made on the anterior vaginal wall, and the dissection was stopped when the index finger inserted through the incision, entered in 
contact with the internal surface of the ischiopubic bone.

(ii) Subsequently, a $5 \mathrm{~mm}$ vertical skin incision was made on the internal surface of the thigh, $10 \mathrm{~mm}$ above a horizontal line, indicating the level of the urethral meatus, and $10 \mathrm{~mm}$ outside the ischiopubic ramus.

(iii) Then, a curved tunneling tool was introduced through the obturator foramen from outside to inside, perforating the obturator membrane as it entered and was in contact with the index finger of the contra-lateral hand. Visual checks were conducted in order to control the tunneling device from perforating the lateral vaginal sulcus.

(iv) Finally, the TOT tape was inserted in the eye of the tunneling tool and passed backward through the obturator foramen by removing the device. The tape was placed in a $\mathrm{V}$ shape in a transverse frontal plane between the mid-urethra and anterior vaginal wall without any tension.

Post-operative follow up were done after 1 month, 3 month, 12 month and annually, thereafter. The assessment includes physical examination, history of LUT symptoms and cough stress test (Attachment 2). The post-operative cure is indicated by the disappearance of SUI symptoms and the negative cough stress test.

The cough stress test was performed as follows:

(i) The patients were placed in the supine position, and their bladders were filled with $300 \mathrm{ml}$ of fluid.

(ii) They were then asked to cough in order to observe if there is any leakage noted which would indicate a positive cough stress test.

A specially trained nurse made further follow-up assessment through telephone surveys using questionaires (Attachment 3). Patients who did not require any further continence surgery and who responded "no" to the question of leaking urine during physical activity, coughing, or sneezing were regarded as being successfully treated.

\section{Definitions}

For the purpose of this study the following definitions were used based on International Urogynecology Association Terminology 2010:

(1) SUI is involuntary leakage on effort or exertion or on sneezing or coughing.

(2) Urinary urgency is "the complaint of a sudden compelling desire to pass urine which is difficult to defer.

(3) UUI is complaint of involuntary loss of urine associated with urgency.

(4) MUI is complaint of involuntary loss of urine associated with urgency and also with effort or physical exertion or on sneezing or coughing.

(5) $\mathrm{OAB}$ syndrome is urinary urgency, usually accompanied by frequency and nocturia, with or without urgency urinary incontinence, in the absence of urinary tract infection or other obvious pathology.

The data was analyzed by using SPSS version 12 (SPSS Inc. Chicago, Illinois) for both quantitative and qualitative variables. McNemar chi-square test was used to compare pre and post qualitative variables considering a significant level of $P<0.05$.

\section{Results}

79 TOT procedures were performed during the study period. Only 52 cases were available for analysis, nine records were missing and 18 patientes did not respond or were uncontactable. The mean age of the population was $56.9 \pm 10.6$ years (range, $41-78$ years). As it is well known that an increase in body weight results in increase likelihood of urinary incontinence, patients BMI were also recorded. 21 subjects were deemed overweight, 19 obese, and the remaining were normal weight. None of the patients had previous incontinence surgery. Mean follow up was 19 months (range, 6-34 months). Patient demographic data is listed in table 1. 
Table I: Patients Characteristics

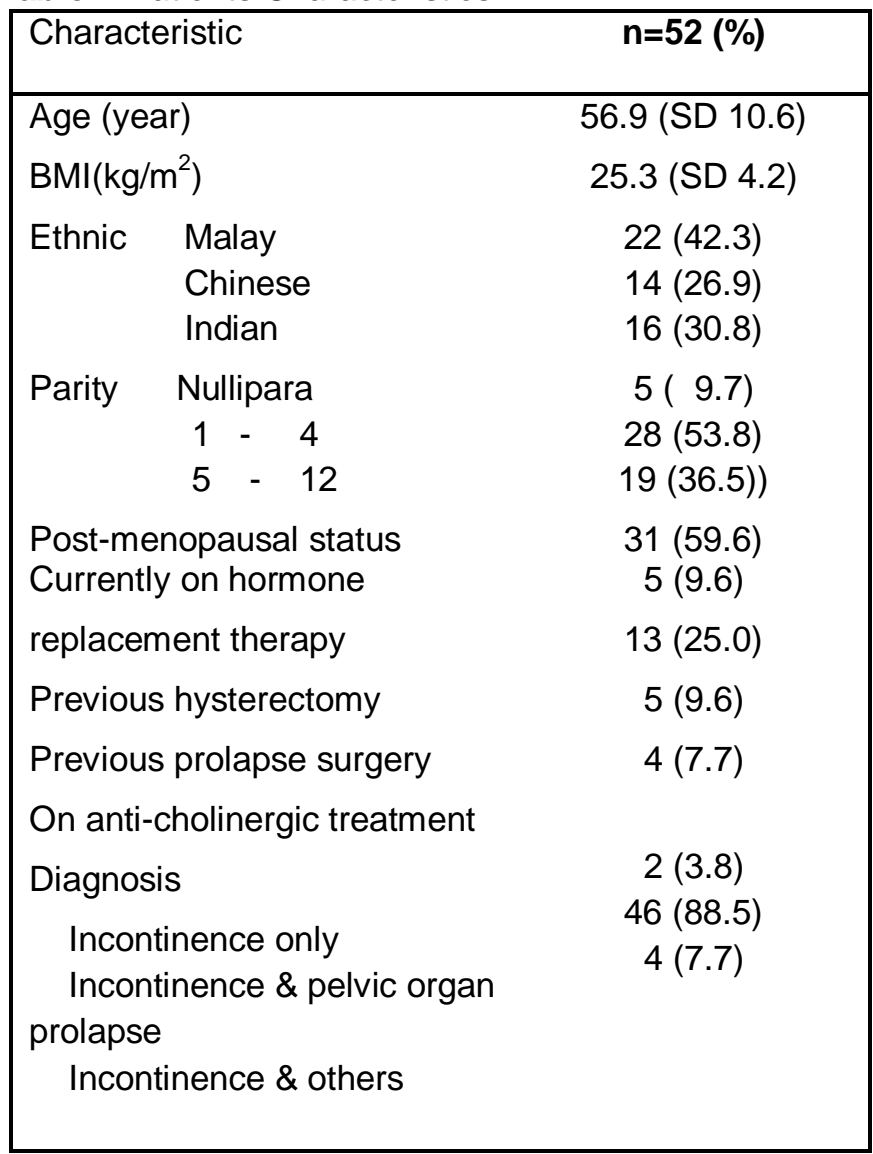

Others: hooded clitoris, deficient perineal body and hemorrhoid.

The urodynamic stress incontinence (USI) was confirmed by filling cystometry. In the majority of patients $(n=21: 40.4 \%)$ had to use pad, $6(11.5 \%)$ leaked during sexual intercourse and wet bed. In most cases $(n=46$ : $88.5 \%$ ) had concurrent pelvic organ prolapse. Twenty four cases (46\%) had pre-operative urgency and frequency which indicates $O A B$ and $16(30.8 \%)$ had MUI. Voiding problems were noted in 26 cases (Table $3)$. Five $(9.6 \%)$ had TOT procedure only, while 38 $(88.5 \%)$ had concomitant vaginal hysterectomy and/or anterior/posterior colporrhaphy.

There was no bladder or major blood vessel injury during the procedures (Table 2). Post-operative complication was found in $19 \%$ of patients. One patient had to return to operating theatre (OT) within 24 hours because of acute urinary retention. The tape was then readjusted to release the obstruction. Partial division of
Table 2: Post-Operative Complications

\begin{tabular}{|l|c|}
\hline & Frequency (\%) \\
\hline UTI & $1(1.9)$ \\
Prolonged IDC & $3(5.8)$ \\
Return to OT & $1(1.9)$ \\
De novo urgency & $3(5.8)$ \\
Erosion & $1(1.9)$ \\
Hematoma & $1(1.9)$ \\
Wound infection & $1(1.9)$ \\
Bladder perforation & 0 \\
\hline IDC = indwelling catheter & \\
OT = operating theatre & \\
UTI = urinary tract infection &
\end{tabular}

the tape was carried out in two (3.6\%) cases, one (1.9\%) required urethral dilatation, tape erosion occurred in one case $(1.9 \%)$. Unfortunately, one case had to undergo total excision of the tape due to persistent urinary retention. However it was later found to be the result of bladder failure. Although her pre-operative uroflowmetry revealed straining pattern, the maximum flow rate $(Q$ $\max$ ) was observed to be normal, $17.1 \mathrm{ml} / \mathrm{s}$.

Majority of patients with preoperative $O A B$ and voiding symptoms were successfully treated $(p<0.001)$. Results are summarized in table 3 and 4 . Despite great improvement in $\mathrm{OAB}$ symptoms, three cases developed de novo urgency. Forty-two (80.7\%) cases were completely dry, confirmed through the use of cough stress test. Subjective assessment via telephone interview revealed that $49(94.2 \%)$ patients were satisfied with the procedure. Continence rate over the last week was higher of which 45 (86.5\%) patients were completely dry while the rest required pad protection. A review of 10 cases which SUI symptoms did not resolved, showed that all except one patient were of the post-menopausal group (mean age $=67 \pm 11.2 \mathrm{yrs}$ ) and, were associated with pelvic organ prolapse and MUI. Their mean BMl was $25.6 \pm 4.9 \mathrm{~kg} / \mathrm{m}^{2}$ and two women had previous history of cystocele repair and vaginal hysterectomy. 
Table 3: Lower Urinary Tract Symptoms at Mean 19 Months Follow Up

\begin{tabular}{|c|c|c|c|c|}
\hline \multirow[t]{2}{*}{ *Symptom } & \multirow[t]{2}{*}{ Pre operative $n=52$} & \multicolumn{3}{|c|}{ Post operative $\mathrm{n}=52(\%)$} \\
\hline & & Cured & improved & failed \\
\hline SUI & 52 & $42(80.7)$ & $7(13.5)$ & $3(5.8)$ \\
\hline Mixed UI & 16 & $6(37.4)$ & $7(43.8)$ & $3(5.8)$ \\
\hline Frequency & 24 & $17(70.8)$ & $6(25.0)$ & $1(4.2)$ \\
\hline Urgency & 24 & $13(54.2)$ & $9(37.5)$ & $2(8.3)$ \\
\hline Voiding difficulty & 13 & $10(76.9)$ & $2(15.4)$ & $1(7.7)$ \\
\hline Incomplete empty & 16 & $13(81.2)$ & $1(6.3)$ & $2(12.5)$ \\
\hline
\end{tabular}

${ }^{*}$ Patients can have more than one symptom.

Table 4: Lower Urinary Tract Symptoms Before and After TOT Procedure

\begin{tabular}{|lccc|}
\hline & Pre-Opn (\%) & Post-Opn (\%) & $P^{*}$ \\
\hline SUI & $52(100)$ & $10(19.20)$ & $0.002^{\star *}$ \\
Mixed UI & $16(30.76)$ & $13(25.00)$ & $<0.001$ \\
Frequency & $24(46.15)$ & $7(13.46)$ & $<0.001$ \\
Urgency & $24(46.15)$ & $11(21.15)$ & $<0.001$ \\
Voiding difficulty & $13(25.00)$ & $3(5.76)$ & $<0.001$ \\
Incomplete empty & $16(30.76)$ & $3(5.76)$ & $<0.001$ \\
\hline
\end{tabular}

${ }^{*}$ McNemar Test, ${ }^{* *}$ Exact test

\section{Discussion}

Two systemic reviews which examined the effectiveness of transobturator tape revealed that the objective treatment rate to be between $84 \%$ to $98 \%[12$, 4]. This was found to be higher to that found in our study i.e. $80.7 \%$ only. The difference is likely because of our study population had higher percentage of $\mathrm{MUI}$, were post-menopausal, aged and with concurrent pelvic organ prolapse.

Predictive factors that could affect the efficacy of MUS procedure have been specifically designed by few studies [13-17] which included inexperienced surgeon [13], general anesthesia [13], previous incontinence surgery [15], aging [16,18], medical comorbidities [17], urgency incontinence[17], high grade of cystocele, transobturator approach [17], and concomitant prolapse surgery [19].

Anger et al [20] retrospectively reviewed the records of 1,356 cases of sling procedures of which 467 had concomitant prolapse surgery. At 1 year follow up, the findings suggested that addressing prolapse at the time of sling surgery may avoid an early repeat operation for both SUI and prolapse but with a higher rate of outlet obstruction. However, this study did not provide the severity of incontinence, prolapse or outlet obstruction and type of sling used. Conversely, Barber et al [19] in a clinical trial involving 162 cases of TOT and TVT concluded that concurrent prolapsed surgery and preoperative anti-cholinergic medication used are associated with increased risk of developing recurrent urinary incontinence. Furthermore, increasing age is a factor that specifically related to recurrent of SUI symptoms at 1 year follow up. These findings were consistent with Stress Incontinence Surgical Treatment Efficacy (SISTER) trial, which finds that the significant predictive factors for recurrence of SUI [21] are related to the severity of concurrent urgency incontinence symptoms, prolapse stage and being post- menopausal without hormone therapy. This trial prospectively evaluated the risk factors for treatment failure after Burch colopsuspension and rectus fascia sling procedures.

Few authors suggested that prolapse surgery has a negative impact on the anti-incontinence surgery by causing urethral denervation resulting from vaginal dissection, or because women with both pelvic organ prolapse (POP) and SUI represent a group with more severe pelvic floor dysfunction than those with SUI alone 
[22]. Whether staging the procedures for women with both POP and SUI i.e. performing prolapse surgery first followed by anti-incontinence procedure would have produced better clinical outcomes remains unclear.

Eighty eight percent of our study population had undergone concomitant prolapse surgery. This may explain why significant improvement in voiding problems (voiding difficulty and incomplete empty) after operation. There was one case that had recurrence of SUI after 2 years of surgery. There was no case of recurrent prolapse at median follow up of 19 months. It is therefore unclear as to whether concomitant MUS procedure reduces the risk of prolapse recurrence because there were only 5 women who only had TOT procedure. It is interesting to note that cases who failed TOT procedure were not obese. The reason of failure was most probably related to concurrent prolapse procedure, old age/postmenopausal and mixed type of incontinence.

OAB symptoms were significantly cured following MUS procedure which was consistent with our previous study and supported by others [23, 24]. Those with symptom of frequency had the highest cure $70.8 \%$ (16) with MUI being the lowest 37.5\% (6). Duckett et al [23] found that $63 \%$ of his study subjects were cured from their irritable bladder symptoms when assessed with a quality-of-life questionnaire. The reason why MUS cures detrusor overactivity (DO) is unclear. Involuntary detrusor activity is induced by urethral afferent nerve. Dissection during placement of SUS might damage this neural pathway [24] making DO disappeared. However, MUS cannot be advocated as a main treatment of $O A B$ except for those who failed medical therapy.

The limitation of this study is in its small sample size due to limited number of selective cases recruited in our centre, especially in light that SUI only problem were needed to reduce the cofounding factors in the final analyses.

\section{Conclusion}

The efficacy for SUI using TOT is lower as compared to TVT especially in cases with concomitant prolapse and urgency incontinence. TOT procedures significantly improves $O A B$ symptoms with high patient's acceptance rate and low complications.

\section{Acknowledgements}

We would like to thank Associate Professor $\mathrm{Dr}$ Jamalludin Ab Rahman, Department of Public Health and Dr Azha Syaril for their contribution.

\section{References}

1. Nilsson CG, Kuuva N. The tension-free vaginal tape procedure is successful in the majority of women with indications for surgical treatment of urinary stress incontinence. Br J Obstet Gynaecol 2001; 108:414-419

2. Ulmsten U, Petros P. Intravaginal slingplasty (IVS): an ambulatory surgical procedure for treatment of female urinary incontinence. Scand J Urol Nephrol 1995; 29:75-82

3. Bemelmans BL, Chapple CR. Are slings now the gold standard treatment for the management of female urinary stress incontinence and if so which technique? Curr Opin Urol 2003; 13:301- 307.

4. Sung VW, Schleinitz MD, Radin CR, et al. Comparison of retropubic vs transobturator approach to midurethral slings: a systematic review and meta-analysis. Am J Obstet Gynecol 2007; 197:3-11

5. Ulmsten $\mathrm{U}$, Johnson $\mathrm{P}$, Rezapour M. A three-year follow up of tension free vaginal tape for surgical treatment of female stress urinary incontinence. $\mathrm{Br} \mathrm{J}$ Obstet Gynaecol 1999; 106:345-350

6. Debodinance $\mathrm{P}$, Delporte $\mathrm{P}$, Engrand JB,et al. Tension-free vaginal tape (TVT) in the treatment of urinary stress incontinence: 3 years experience involving 256 operations. Eur J Obstet Gynecol Reprod Biol 2002; 105:49-58 
7. Meschia $M$, Pifarotti $P$, Bernasconi $F$ et al. Tensionfree vaginal tape: analysis of outcomes and complications in 404 stress incontinent women. Int Urogynecol J Pelvic Floor Dysfunct 2009; 12(Suppl 2):S24-S27

8. Delorme E. Transobturator urethral suspension: mini invasive procedure in the treatment of stress urinary incontinence in women. Prog Urol 2001; 1:13061313

9. Liapis A, Bakas $P$, Creatsas G. Long-term efficacy of tension-free vaginal tape in the management of stress urinary incontinence in women: efficacy at 5and 7-year follow-up. Int Urogynecol J Pelvic Floor Dysfunct 2008; 9:1509-1512

10. Delorme E, Droupy S, de Tayrac R, et al. Transobturator tape (Uratape): a new minimallyinvasive procedure to treat female urinary incontinence. Eur Urol 2004; 45 (2):203-207

11. Naidu A, Lim YN, Barry C et al. Trasobturator tape for stress incontinence: The North Quensland experience . ANZJOG 2005; 45(5): 446-449

12. Novara G, Ficcara V, Boscolo-Berto $R$, et al. Tension-free midurethral slings in the treatment of female stress urinary incontinence: a systematic review and meta-analysis of randomized controlled trial of effectiveness. Eur Urol 2007; 52:663-79.

13. Koops SE, Bisseling TM, van Brummen $\mathrm{HJ}$ et al. What determines a successful tension-free vaginal tape? A prospective multicenter cohort study: results from the Netherlands TVT database. $A m \mathrm{~J}$ Obstet Gynecol 2006; 194:65-74

14. Lee KS, Choo MS, Doo CK et al. The long term (5years) objective TVT success rate does not depend on predictive factors at multivariate analysis: a multicentre retrospective study. Eur Urol 2008; 53:176-182.

15. Chen HY, Yeh LS, Chang WC et al. Analysis of risk factors associated with surgical failure of inside-out transobturator vaginal tape for treating urodynamic stress incontinence. Int Urogynecol J Pelvic Floor Dysfunct 2007; 18:443-447

16. Cetinel B, Demirkesen O, Onal B et al. Are there any factors predicting the cure and complication rates of tensionfree vaginal tape? Int Urogynecol J Pelvic Floor Dysfunct 2005; 15:188-193

17. Paick JS, Cho MC, Oh SJ et al. Factors influencing the outcome of mid urethral sling procedures for female urinary incontinence. J Urol 2007; 178:985-9

18. Paick JS, Oh SJ, Kim SW et al. Tension-free vaginal tape, suprapubic arc sling, and transobturator tape in the treatment of mixed urinary incontinence in women. Int Urogynecol J Pelvic Floor Dysfunct 2008; 19:123-129

19. Barber MD, Kleeman S, Karram MM et al. Risk factors associated with failure 1 year after retropubic or transobturator midurethral slings. Am $\mathrm{J}$ Obstet Gynecol 2008; 199(666):e1-7

20. Anger TJ, Litwin MS, Wang $Q$, et al. The effect of concomitant prolapse repair on sling outcomes. $J$ Urol 2008; 180:1003-1006

21. Brubaker L, Rickey L, Xu Y, Markland A et al. Symptoms of combined prolapse and urinary incontinence in large surgical cohorts. Obstet Gynecol. 2010 Feb; 115(2 Pt 1):310-6.

22. Daneshgari F, Moore $\mathrm{C}$, Frinjari $\mathrm{H}$, et al. Patient related risk factors for recurrent stress urinary incontinence surgery in women treated at a tertiary center. J Urol 2006; 176:1493-9.

23. Duckett JR, Tamilselvi A. Effect of tension-free vaginal tape in women with a urodynamic diagnosis of idiopathic detrusor overactivity and stress incontinence. BJOG 2006; 113:30-33

24. Jung SY, Fraser MO, Ozawa $\mathrm{H}$, et al. Urethral afferent nerve activity affects micturation reflex, implication for the relationship between stress incontinence and detrusor instability. $J$ Urol 1999:162(1):204-212 


\section{Attachment 1}

The MONARC Sling System Evaluation Study (Preop Medical Audit)

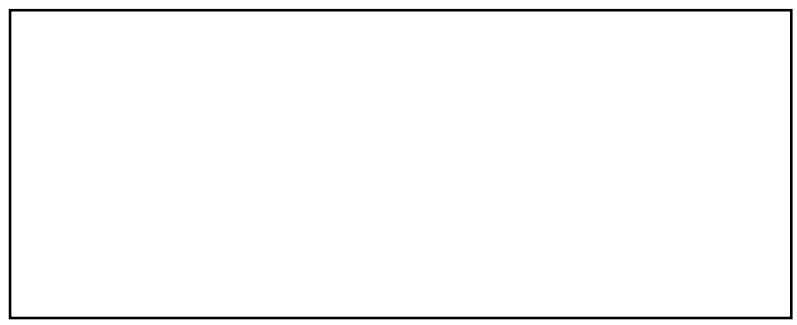

Hospital:

Height(cm):

Weight(kg):

Preoperative Symptoms: (Please circle appropriate answers)

Stress Incontinence: yes / no Urge Incontinence: yes / no

Frequency ( $>10$ daytime or $>2$ overnight). $\quad$ yes / noUrgency: yes / no

Voiding Difficulty: yes / no Incomplete Emptying: yes / no Filling Pain: yes / no

Recurrent UTI (>4/year) yes / no

(Please request patient to fill in Incontinence Impact Questionnaire IIQ-7)

Medical Background:

Premenopausal yes/no Postmenopausal yes / no

HRT yes/no Topical Oestrogen yes/no Anticholinergic yes / no

Past History: (please circle if applicable)

Hysterectomy

Prolapse Repair, please specify type if known:

Incontinence Surgery, please specify type if known:

Obstetric History:

Nulliparous

Multiparous

Preop 1 Hour Pad Test Result: g (Please request patient to perform if not already done)

Preop Urodynamics Results:

Pre CMG Voided Volume: ..ml,

Qmax: $\mathrm{ml} / \mathrm{s}$,

Qave: $. . \mathrm{ml} / \mathrm{s}$

MUCP . $\mathrm{cmH} 2 \mathrm{O}$

Postvoid Residual Volume: ..ml Cystometric Capacity mls

Urodynamic Stress Incontinence Detrusor Overactivity

Dysfunctional Voiding 


\section{Attachment 2}

MONARC Postoperative Evaluation Form (Medical Audit)

Hospital:

Duration since surgery:

Please ask patient to fill in Monarc Operation Follow up Questionnaire and Incontinence Impact Questionnaire (IIQ-7) Post-discharge Complications:

UTI: yes / no Prolonged IDC (>2 days) yes / no

Return to Theatre: yes / no (if yes, please state reason, eg. sling adjustment)

Other complication yes / no (if yes, please specify, eg. DVT)

Objective Findings:

Sling protrusion/erosion: yes / no

LUT symptoms:

Others: (Please specify)

Cough stress Test Result: 


\section{Attachment 3}

\section{MONARC Sling Operation Follow Up Questionnaire}

Name:

Date of assessment:

Time Since MONARC Operation:

\section{Please circle the appropriate answer for each of the following questions.}

1) Urinary stress incontinence (urine leakage when you cough, sneeze, conduct physical activity)

Present before the operation: yes / no

Do you have this symptom now: yes / no

If yes, how does it compare to before the operation?

Better / same / worse

2) Urinary frequency (having to empty your bladder very often)

Present before the operation: yes / no

Do you have this symptom now: yes / no

If yes, how does it compare to before the operation?

\section{Better / same / worse}

3) Urinary urgency (sudden onset strong, compelling desire to empty your bladder)

Present before the operation: yes / no

Do you have this symptom now: yes / no

If yes, how does it compare to before the operation?

Better / same / worse

4) Urinary leakage as a result of urinary urgency (i.e. not getting to toilet in time)

Present before the operation: yes / no

Do you have this symptom now: yes / no

If yes, how does it compare to before the operation?

Better / same / worse

5) Difficulty emptying your bladder (eg. having to strain to pass urine, difficulty getting started, poor urine stream)

Present before the operation: yes / no

Do you have this symptom now: yes / no

If yes, how does it compare to before the operation?

Better / same / worse

6) The bladder does not feel empty after passing urine (eg. When you stand up you start dribbling some urine, need to void again within 5-10 minutes)

Present before the operation: yes / no

Do you have this symptom now: yes / no

If yes, how does it compare to before the operation?

Better / same / worse

7) Pain or discomfort in the lower abdominal or groin area

Present before the operation: yes / no 
Do you have this symptom now: yes / no

If yes, how does it compare to before the operation?

\section{Better / same / worse}

8) Over the last week, how would you rate your continence?

(a) Completely dry

(b) Substantially continent: may have periodic leakage of small amounts, but additional protection is not needed

(c) Some protection may be required: needing up to 3 pads per day, or when leakage occur not more than once weekly

(d) Substantially or totally incontinent: requiring more than 3 pads a day, or large leak 2 or more times a week

9) Are you satisfied with the result of the operation? Yes / no

10) Any additional comments? 